\title{
Investigar la Comunicación en el Departamento de Administración de Empresas y Marketing de la Universidad de Sevilla
}

Francisco J. Caro González (Área de Organización de Empresas)

Carlos J. Rodríguez Rad (Área de Marketing)

http://dx.doi.org/10.12795/AdMIRA.2009.01.18

Resumen: Este trabajo recoge las principales investigaciones sobre comunicación llevadas a cabo por los profesores del Departamento de Administración de Empresas y Marketing. Se ha hecho una revisión de las publicaciones de los últimos 20 años y se han extraído y agrupado por tópicos los trabajos de investigación realizados. Se ha tenido en cuenta la pertenencia a las dos áreas específicas del Departamento "Organización de Empresas" y "Marketing". Este estudio pone de manifiesto la importancia de la comunicación en la investigación de administración de empresas y la involucración del profesorado adscrito a la Facultad de Comunicación, que centran parte de su labor investigadora en los conocimientos específicos que se imparten en el centro.

Palabras clave: investigación, publicidad, comunicación, marketing, organización de empresas.

Key words: Research, advertising, communication, marketing, management.

\section{La comunicación en Administración de Empresas}

Las organizaciones se han convertido en agentes sociales comprometidos con su ambiente, al que deben respetar, favorecer e incluso dar ejemplo con una actuación ética y solidaria ${ }^{1}$. Todo esto desemboca en una intensa actividad comunicativa. Además, la actual situación competitiva de muchos sectores hace indispensable que la empresa busque una diferenciación no sólo basada en la imagen de sus productos y servicios, sino en la imagen global de la propia empresa, que muchas veces actúa en actividades diversas y precisa de una identidad global que permita su expansión y diversificación.

Las organizaciones actuales son realidades complejas compuestas por multitud de subsistemas y las informaciones que estos producen pueden ser a veces contradictorias, por lo que es preciso homogeneizar todos los mensajes que fluyen de la misma con el fin de reflejar una identidad coherente y acorde con su misión. Esto sólo será posible si

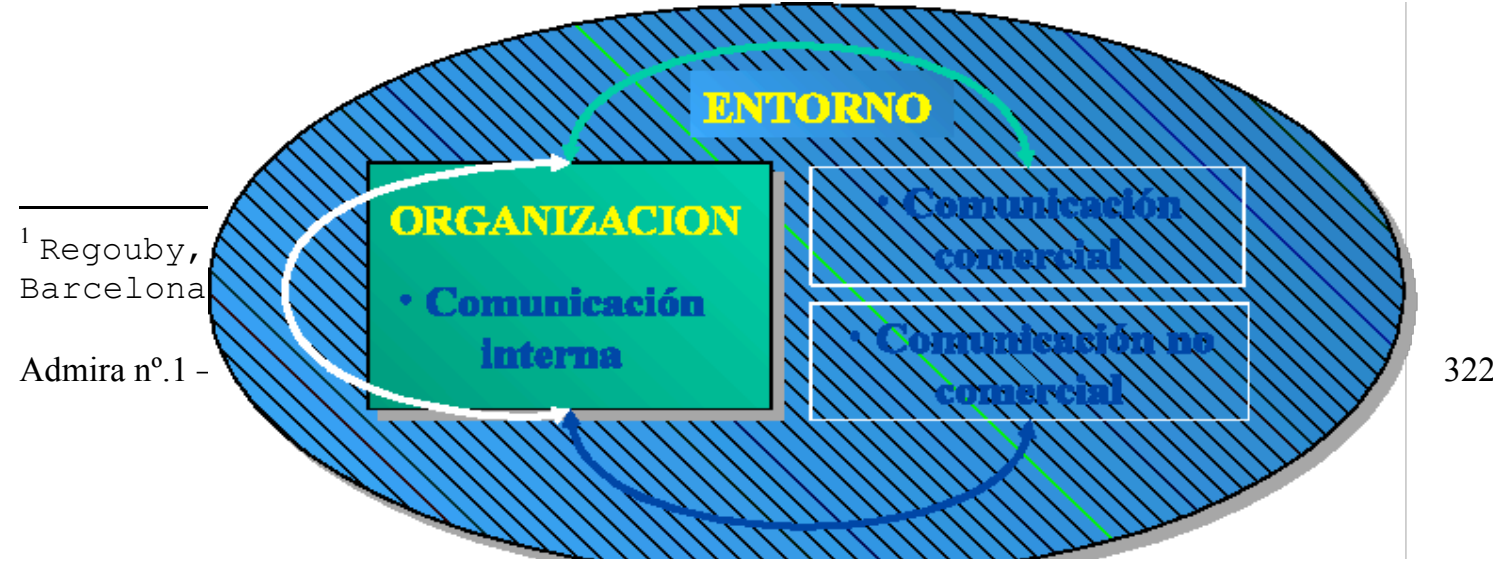


se produce una coordinación, a través de una estrategia de comunicación, de las distintas fuentes comunicativas.

La comunicación global de la organización pretende integrar las diferentes comunicaciones que fluyen en la empresa. Según el destino de la comunicación podemos distinguir ${ }^{2}$ :

\begin{tabular}{|l|l|l|}
\hline \multicolumn{2}{|l|}{ COMUNICACIÓN EXTERNA: } & $\begin{array}{l}\text { COMUNICACIÓN } \\
\text { INTERNA. }\end{array}$ \\
\hline COMUNICACIÓN COMERCIAL & COMUNICACIÓN NO COMERCIAL & - Personal de la empresa \\
\hline Comunicación con el gran público: & Comunicación de reclutamiento: & - Dirección \\
- Consumidores & - Demandantes de empleo & - Accionistas \\
- Prescriptores & - Escuelas y Universidades & - Filiales \\
Comunicación Profesional: & Comunicación Financiera: & - Grupo \\
- Distribuidores & - Medios financieros & \\
- Profesionales del sector & - Medios Bursátiles & \\
Comunicación "business to & Comunicación política: & \\
business" & - Poderes públicos & \\
- Empresas clientes y proveedores & - Administraciones & \\
& - Colectividades locales & \\
& Comunicación Social: & \\
& - Sindicatos & \\
& - Asociaciones & \\
& Comunicación de opinión pública: & \\
& - Ciudadanos en general & \\
\hline
\end{tabular}

Es evidente que todos los tipos de comunicación, tanto externa como interna, deben de estar al servicio de la misma causa, pero aunque ambas empleen soportes y técnicas semejantes, los mensajes internos y externos no pueden tener el mismo talante.

La comunicación externa trata de ubicar a la empresa en un mercado, dotarla de una personalidad reconocible para todos los públicos y de una identidad distinta $\mathrm{y}$ diferenciada.

\footnotetext{
${ }^{2}$ Villafañe, J.(1993). Imagen Positiva. Ed. Pirámide, Madrid.
} 
La comunicación interna se emplea como un instrumento estratégico para mejorar las relaciones entre los miembros de la organización, posibilitar su integración y favorecer la existencia de una cultura compartida entre todos.

La importancia que ha ido adquiriendo la comunicación en las organizaciones ha facilitado su implantación como función específica en la empresa, una función asignada a un responsable, que en muchas ocasiones ha propiciado la modificación de la estructura organizativa mediante la creación de un departamento propio.

Las dos áreas sobre las que se investiga e imparte docencia en el Departamento de Administración de Empresas y Marketing de la Universidad de Sevilla son "Organización de Empresas" y "Comercialización e Investigación de Mercados (Marketing)".

La comunicación en el área de organización se investiga como una función continua que llevan a cabo los directivos y que afecta al resto de las funciones administrativas, tanto a las continuas como a las secuenciales. Por ello podemos encontrar trabajos que investigan el papel de la comunicación en la planificación, en la organización, en la dirección (liderazgo y motivación) o en el control. También se estudia como un elemento esencial de la gestión de los recursos humanos.

En el área de Marketing la comunicación también es un tema de estudio muy relevante. La comunicación es una de las variables de la mezcla de marketing por lo que se ha analizado como una herramienta esencial para situar las organizaciones en la mente de sus consumidores con atributos positivos.

Por otra parte, los profesores del Departamento han llevado a cabo investigaciones concretas en las empresas de comunicación, con el objeto de comprender estas organizaciones o de aprovechar sus singularidades para estudiar tópicos clásicos en la disciplina: cambio organizativo, liderazgo, comportamiento estratégico, género, etc.

Por último también queremos incorporar a este trabajo las aportaciones que los profesores del departamento han hecho innovando en la enseñanza de las asignaturas relacionadas con las licenciaturas de Periodismo y Publicidad y Relaciones Públicas en las que imparten docencia.

El proceso de trabajo ha consistido en extraer de SISIUS (http://investigacion.us.es/sisius/sis_dep.php?id_dpto=1) las fichas de los profesores del 
Departamento y analizar en sus investigaciones aquellas relacionadas con la comunicación. Hemos tratado de ser lo más meticulosos posible en la selección de los trabajos, si en algún caso hemos omitido algún trabajo por no estar disponible en la base de datos o haber sido incorrectamente analizado pedimos disculpas. Hemos agrupado los trabajos en grandes tópicos de investigación. Las investigaciones recogen sobre todo publicaciones en libros, capítulos de libros, revistas y comunicaciones presentadas en congreso. En el caso de organización hemos dejado fuera de esta selección las abundantes y excelentes publicaciones sobre sistemas de información por considerar que son aspectos más propios de la Economía de la Empresa y alejados de los intereses de los investigadores de la Comunicación.

El número de investigaciones relacionadas con la comunicación durante el periodo 1989-2008 lo tenemos reflejado en la siguiente tabla. Podemos observar el crecimiento de su número con la creación de la Facultad.

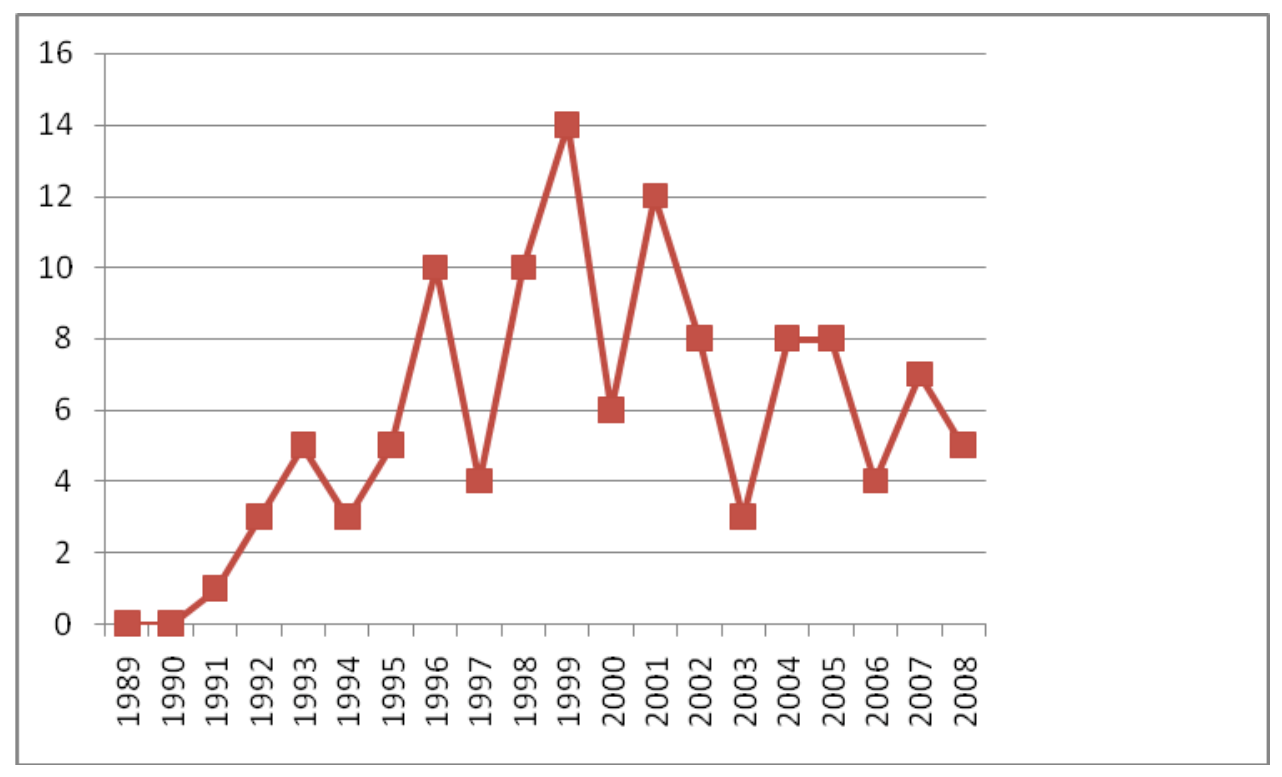

Ilustración 1: Evolución del número de investigaciones en comunicación

La estructura de categorías profesionales del departamento puede ser observada en la siguiente tabla:

\begin{tabular}{|l|l|}
\hline \multicolumn{2}{|c|}{ PROFESORES DEL DEPARTAMENTO POR CATEGORÍA } \\
\hline CATEDRÁTICOS & 10 \\
\hline T.U. & 24 \\
\hline
\end{tabular}




\begin{tabular}{|l|l|}
\hline T.E.U. & 25 \\
\hline CONTRATADOS DOCTORES & 7 \\
\hline AYUDANTES LOU & 3 \\
\hline ASOCIADOS & 11 \\
\hline COLABORADORES & 9 \\
\hline TOTAL & 99 \\
\hline
\end{tabular}

Es llamativo el hecho de que los diez profesores que imparten o han impartido docencia en la Facultad de Comunicación son autores o coautores del $48 \%$ de las investigaciones que forman parte de este estudio. Este dato revela la implicación del departamento en la especificidad de los conocimientos que se imparten en el centro.

\section{ORGANIZACIÓN DE EMPRESAS}

Desde los inicios de la Facultad de Comunicación, el Departamento de Administración de Empresas y Marketing ha impartido los conocimientos relacionados con la gestión de las empresas del sector en comunicación. Estos conocimientos se han estructurado en la asignatura troncal de segundo ciclo de la Licenciatura de Periodismo "Empresa Informativa" (4'5 créditos) y la obligatoria, de último curso de la Licenciatura de Publicidad y Relaciones Públicas "Organización y Gestión de Empresas de Servicios” (9 créditos). Al mismo tiempo, se ofrecían asignaturas de libre configuración muy atractivas para los estudiantes de la facultad: "Creación de Empresas para Estudiantes sin formación empresarial" (impartida en la propia Facultad de Comunicación), "La enseñanza de administración de empresas a través del cine" o "Emprendedores y cine". Por otro lado los profesores del área han participado muy activamente en la docencia de los cursos de postgrado ofertados desde la Facultad de Comunicación: Máster en Gestión de Empresas Audiovisuales, Curso de Expertos en Periodismo Local, Máster en Dirección de la Comunicación Empresarial e Institucional, o los cursos de doctorado del Departamento de Periodismo II. El esfuerzo de los profesores por adaptar los conocimientos de la Economía de la Empresa a las especificidades de las licenciaturas de la comunicación ha dado lugar a numerosos trabajos académicos. 
Las investigaciones relacionadas con la Comunicación realizadas desde el área de organización de empresas son muy variadas y aportan una mayor comprensión, tanto de las empresas de comunicación como de las diferentes variables comunicativas aplicadas a la función administrativa.

Por ello hemos estructurado este apartado en tres grandes bloques: empresas de comunicación, la comunicación como herramienta de gestión y la docencia en la Facultad de Comunicación.

\section{INVESTIGAR LA EMPRESA DE COMUNICACIÓN}

Un trabajo temprano sobre el sector de la empresa informativa es el publicado por Caro y Rodríguez (1995) donde se destaca la singularidad del producto informativo (la información) y se establece una categorización de los tipos de información en función del valor que aporta para la empresa y para las audiencias.

Fruto de ese interés inicial por las empresas informativas se realizan en el departamento diversos estudios para analizar los estilos de dirección de los máximos responsables de las empresas informativas. Traverso y García del Junco (1997) y Caro y García del Junco (1998) realizan un estudio empírico para determinar el estilo de dirección de los directores de los medios de comunicación españoles según el modelo de Likert, al mismo tiempo comprueban la validez de los roles administrativos definidos por Mintzberg en los directivos del sector de la prensa escrita.

En 1999, los profesores Rodríguez-Bobada y Castellanos (1999), en un esfuerzo prospectivo y ante los cambios que se anuncian de cara al nuevo siglo (XXI) analizan el mercado futuro de la televisión para el nuevo milenio.

En 2002, el profesor Caro, realiza su tesis doctoral bajo la dirección del Catedrático Emilio Díez. El tema elegido queda resumido en el título de la misma: "Efectos de la Innovación y el Cambio en las Empresas Informativas". Esta investigación centrada en las empresas editoras de prensa andaluzas ha sido el germen de diversos trabajos posteriores: Caro (2002), Caro y Jiménez (2006) y Caro (2007).

A nivel estratégico se ha estudiado el conjunto de la industria de la comunicación. Caro y Oviedo (1996) analizan los procesos de concentración en el sector audiovisual identificando las alianzas, absorciones y fusiones que se están llevando a cabo en España. 
También podemos destacar el manual de Marketing para empresas audiovisuales publicado por Rodríguez (2003) que nace en el seno del Máster en Gestión de Empresas Audiovisuales y trata de llenar un importante vacío existente en ese ámbito.

En 2006, como preámbulo a los cambios previstos con el proceso de Bolonia, Caro y Jiménez publican un trabajo que realiza una radiografía del mercado de trabajo de los periodistas. A partir de información secundaria de diversas fuentes describen la situación del empleo en el sector y de las condiciones en las que los futuros egresados deben obtener su primer empleo.

Tras 16 años de docencia e investigación en el terreno de la empresa informativa el profesor Caro publica un manual (2007) que recoge los conocimientos básicos de la asignatura: "Gestión de Empresas Informativas". Este libro llena el hueco temporal dejado por los primeros manuales en la materia correspondientes al inicio de la década de los 90. "Gestión de Empresas Informativas" está siendo utilizado como manual en diversas facultades de comunicación de España y Latinoamérica.

También la Responsabilidad Social Corporativa ha sido estudiada en las empresas de comunicación. Como consecuencia de diferentes investigaciones sobre la responsabilidad social corporativa realizadas en sectores diversos, Caro (2008) decide realizar un estudio de caso en las empresas de comunicación, y para ello elige la empresa editora de El País, que acababa de experimentar una importante transformación (del producto y organizativa).

En el año 2007, nace en el seno de la Facultad de Comunicación un proyecto de investigación que agrupa a profesores de diferentes áreas de conocimiento que imparten docencia en el centro: organización de empresas, marketing, periodismo y publicidad. Se trata del grupo SANIMUEI (Satisfacción de las Necesidades Informativas de las Mujeres en las Empresas Informativas), cuyo objetivo es analizar la presencia de las mujeres en las empresas informativas. Este grupo presentó un proyecto de investigación a la convocatoria nacional de $\mathrm{I}+\mathrm{d}+\mathrm{i}$ y se le otorgó una subvención del Ministerio de Asuntos Sociales. El proyecto concluirá en 2010. La mayoría de los componentes del grupo ya habían trabajado en temas de género relacionados con las empresas informativas. De esta manera publicaron trabajos sobre la percepción de la mujer como directiva en las empresas informativas: Caro, García, Rodríguez y Jiménez, (2007); y Caro, García, Rodríguez (2007). 
En esta misma línea, y ya constituido el grupo SANIMUEI se realizó una investigación sobre las mujeres emprendedoras en el sector de la comunicación (Caro, Rodríguez y García, 2008). Este campo de estudio sigue abierto dentro del grupo.

La importancia que tiene la comunicación en el área de organización de empresas ha llevado a que los profesores adscritos a la Facultad de Comunicación desempeñen una importante labor divulgativa de sus conocimientos. Para ello han elaborado material docente para las plataformas virtuales: Caro (2008) y Caro y Orti (2008). También son autores de blogs donde exponen sus reflexiones sobre el sector y dan información de actualidad y académica de utilidad para los estudiantes y los profesionales:

http://fcom.us.es/blogs/empresasdecomunicacion/ http://fcom.us.es/blogs/mujeresempresas/

Queremos concluir indicando que la principal línea de investigación de los profesores del área de organización de empresa en este apartado son los estudios de género y el cambio organizativo en las empresas informativas.

\section{COMUNICACIÓN Y DOCENCIA}

Queremos destacar las investigaciones docentes de los profesores del departamento adscritos a la Facultad de Comunicación destinadas a divulgar las innovaciones didácticas aplicadas en sus asignaturas.

En 1993, Caro realiza un estudio sobre el uso de la prensa escrita en la enseñanza de las asignaturas de administración de empresas impartidas en la Facultad de Comunicación.

Más adelante, Caro y Rodríguez (1997 y 1998) publican dos artículos que explican, justifican y valoran una forma singular de utilizar las producciones cinematográficas en la enseñanza de la asignatura de Periodismo "Empresa Informativa". Tras la obtención de un proyecto de innovación docente, elaboran material audiovisual a partir de las películas que reflejan el mundo del periodismo que es utilizado en el aula como ejemplos o como casos prácticos. 
También otros profesores innovan e investigan sobre cómo el cine puede ser utilizando en la enseñanza de administración de empresas: Casanueva et al (2000); Caro et al (2000); y Caro, Casanueva y Rufino (2000). En estos trabajos se exponen las experiencias docentes de los profesores, la metodología y los sistemas de evaluación aplicados. Estas innovaciones fueron merecedoras del premio a la innovación docente de la Universidad de Sevilla del año 2000.

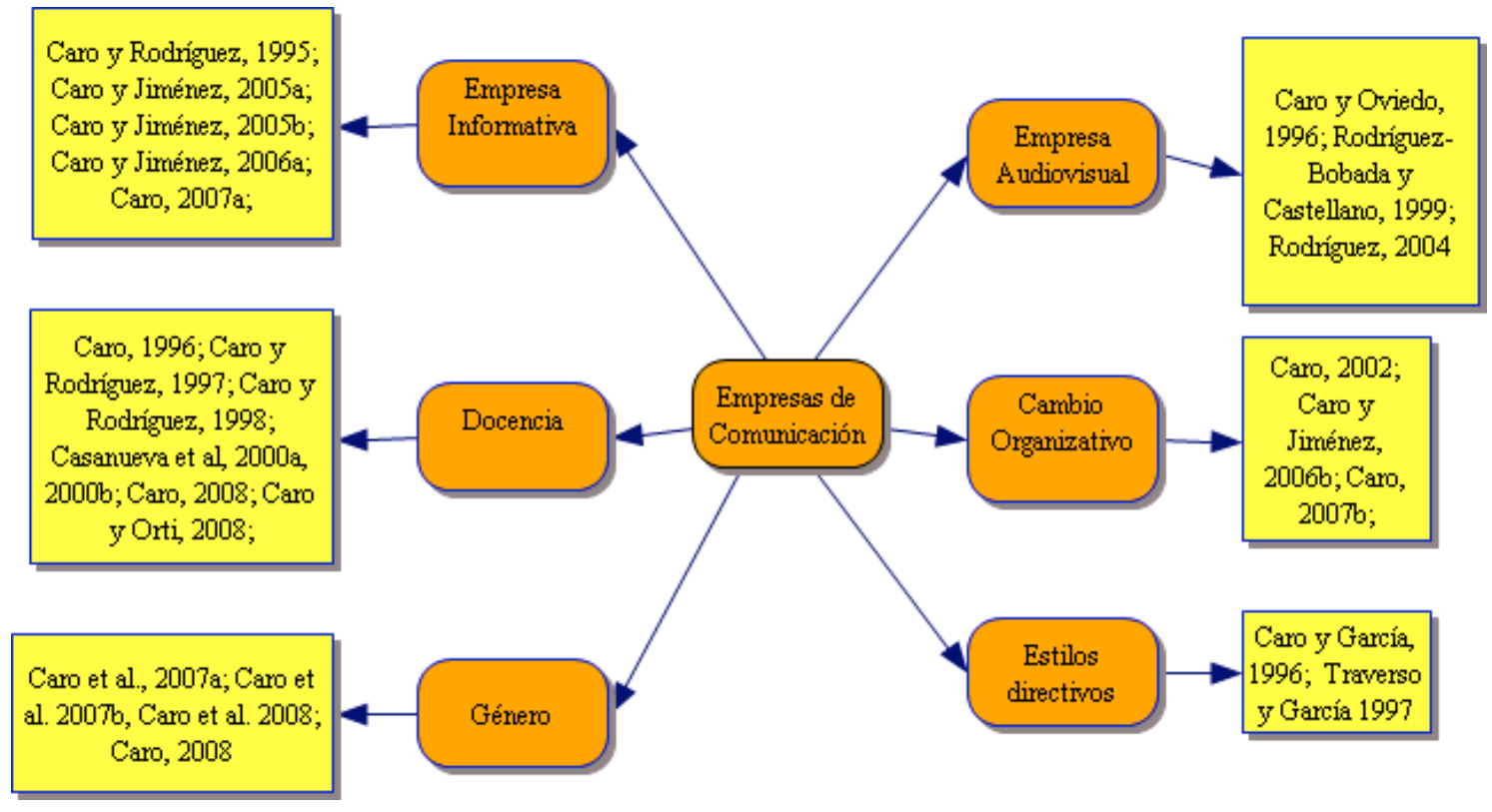

Ilustración 2: Investigaciones relacionadas con las Empresas de Comunicación

\section{LA COMUNICACIÓN COMO HERRAMIENTA DE GESTIÓN}

Otra línea de investigación desarrollada por los profesores del departamento está ligada a la consideración de la comunicación como función administrativa. La comunicación se identifica como una variable que cohesiona la organización, que constituye la argamasa que transforma a la empresa en un sistema integrado. Al mismo tiempo se muestra como una herramienta esencial para la gestión de los recursos humanos y la implantación del cambio en las organizaciones.

Un trabajo pionero es el publicado por Casanueva y Caro (1993) en el que se analiza la situación de la comunicación interna en las empresas españolas. Estos profesores a partir de un estudio empírico de carácter cualitativo y estructurado según el modelo de comunicación de la teoría cibernética exponen como es percibida esta función por los expertos en comunicación. En esta misma línea continúan los profesores 
Calvo y Criado (2001) al estudiar los procesos de comunicación interna en las Universidades mediante la aplicación del benchmarking.

En 1996 un grupo de profesores publican un libro sobre la comunicación como recurso estratégico en las organizaciones (Criado et al, 1996). Y en 1999, las profesoras Cruz y Revilla analizan el papel de la comunicación en los procesos de internacionalización de las empresas.

La comunicación también ha sido estudiada desde la perspectiva de las instituciones, el paso de la comunicación de empresa a la comunicación corporativa o institucional sugiere nuevos temas de investigación. De esta forma, se analiza la importancia de la arquitectura en la creación de identidad e imagen en las organizaciones (Caro, 1996; Jiménez y Caro, 2006). Otro trabajo (Castellanos y Carvajal, 1999) examina la campaña institucional de comunicación en el lanzamiento del euro. Hay que destacar que la mayoría de las investigaciones en esta línea ha sido desarrollada por los profesores del área de marketing por lo que tendrá un mayor desarrollo es ese apartado.

La medición del impacto de las nuevas tecnologías de la información y la comunicación en las organizaciones ha sido otro de los temas que ha preocupado a los profesores del área de Organización. Casanueva, Ganaza y Espasandín (2004) investigan los cambios producidos por estas tecnologías en las empresas andaluzas de economía social. García del Junco (2004) hace lo propio pero en relación a su efecto en los procesos de globalización. La profesora Alves defiende en 2005 una tesis doctoral titulada "La Dirección de los Recursos Humanos y las Nuevas Tecnologías de la Información y la Comunicación (Formación a Distancia y e-Trabajo)" en la que destaca el cambio que se produce en los procesos de formativos y operativos como consecuencia de las innovaciones tecnológicas en comunicación.

En relación a la gestión de recursos humanos hay un grupo de profesores que han centrado su investigación en los anuncios de reclutamiento como soporte de comunicación de la empresa (comunicación con el mercado de trabajo). Entre estos autores cabe destacar a Rubio (2000a, 2000b) que ha descrito los aspectos redaccionales y comunicativos de estos anuncios. Otros trabajos que inciden este canal de comunicación no comercial son: Díaz y Picón (2001), Castro y Cossio (1998) y Oviedo y Quirós (1997). 


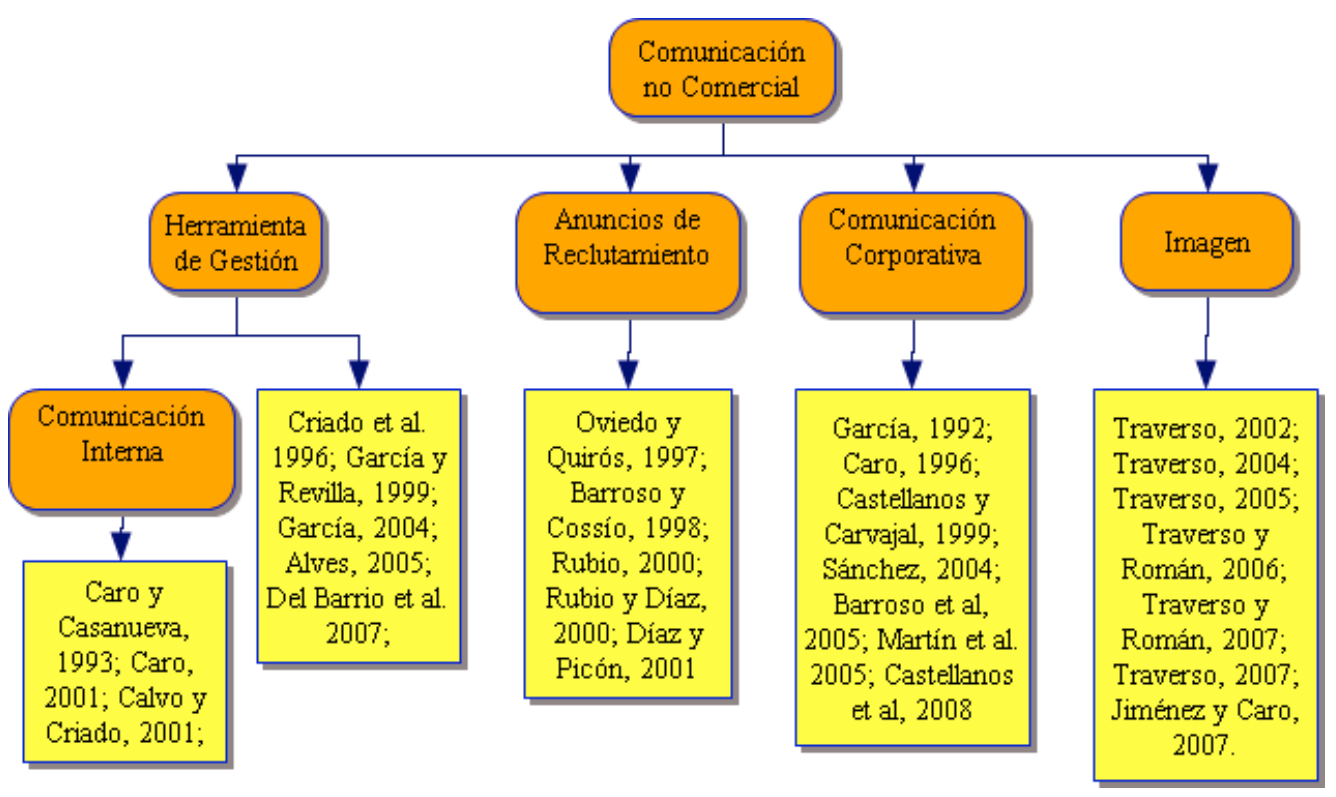

Ilustración 3: Investigaciones relacionadas con la comunicación comercial

\section{MARKETING}

En cuanto al contenido de las investigaciones debemos remarcar que viene poderosamente marcado por la implicación de los profesores del departamento con la licenciatura de Publicidad y Relaciona Públicas, en la única que imparten docencia aquellos que pertenecen al área de Comercialización e Investigación de Mercados (Marketing).

Los resultados de las investigaciones se orientan fundamentalmente a la disciplina de marketing y en su relación con las variables de comunicación comercial y con las empresas del ámbito de la comunicación comercial.

Podemos agrupar los trabajos en dos grandes líneas de la investigación: la publicidad y otras formas de comunicación comercial.

\section{LA PUBLICIDAD}

La vinculación del departamento de Administración de Empresas y Marketing con el ámbito de la publicidad se inicia incluso antes de que naciese en Sevilla la Facultad de Comunicación, así podemos observar que en 1976 Enrique Martín defiende su tesis doctoral "Planificación y Control de Campañas Publicitarias" que intenta encuadrar la publicidad en el seno de la gestión empresarial. 
A finales de los 70 se profundiza en el control publicitario (Martin, 1976) y en la manera satisfactoria de determinar el presupuesto publicitario (Martín, 1979).

En 1980 se publica el libro "Planificación Publicitaria" que ha sido referencia en nuestro país durante muchos años pues tanto para profesionales de la publicidad que diseñan las campañas como para alumnos de diferentes licenciaturas. Ya en 1993 defiende su tesis doctoral el hoy Decano de la Facultad de Ciencias Económicas y Empresariales Javier Landa bajo la dirección de Enrique Diez de Castro que llevó por título: "La Publicidad: la Influencia del Factor Legal en su Gestión".

A partir de entonces han sido numerosos los trabajos publicados relacionados con esta disciplina:

A principios de la década de los 90 Rodríguez Rad publica varios artículos en los que analiza la publicidad desde la perspectiva del marketing "El sector del automóvil: motor de la publicidad", "La adaptación de la publicidad a su nuevo escenario" y “Aspectos de la publicidad en la Comunidad Económica Europea”

Algunos trabajos se han centrado en la relación entre la Universidad y la Publicidad (Traverso, 1995; Sánchez y Rodríguez, 2002) o la incorporación de los egresados de publicidad al mercado laboral (López, Traverso y López)

Los efectos de la incorporación de España a la Comunidad Económica Europea centró la atención de algunos investigadores, así Martín (1992) analiza los efectos de la Globalización del Mercado Europeo sobre la Gestión Publicitaria, y García, Rubio y Rodríguez (1993) los aspectos de la publicidad en la CEE.

El anuncio publicitario también ha ocupado gran parte del interés de los investigadores de nuestra área. Se publican trabajos relativos a la Posición del Anuncio en la Pausa Publicitaria (Sánchez, 1998 y 2001), la duración adecuada del anuncio (Sánchez, 1999), el color del anuncio (1999) y la relación entre el recuerdo y la actitud hacia el anuncio (Sánchez, 1999).

Los anuncios de reclutamiento por sus propias características han requerido un tratamiento específico.

Rubio (1999) defiende su tesis doctoral en la que examina los anuncios de empleo en prensa como forma publicitaria y un año después Rubio y Diaz (2000) se centran en la importancia del diseño de los anuncios de ofertas de empleo en prensa. 
Barroso y Cossio (1998) en el marco del proceso de reclutamiento de la fuerza de ventas, investigan las ofertas de empleo en prensa, Oviedo y Quirós (1997) se centran en el caso español, mientras que Diaz y Picón (2001) lo hacen con el buscador de empleo y su comportamiento en relaciona a las ofertas insertas en los anuncios en prensa.

En relación a los tipos de publicidad, López, Casillas y Villegas (1994) analizan la publicidad conjunta como una forma de cooperación.

Otro trabajo se centra en la importancia de la publicidad a un nivel macroeconómico, así Castellanos y Oviedo (1996) tratan los efectos de la inversión publicitaria en la marcha de la economía

Oviedo (1994) destaca el rol de la mujer en los anuncios publicitarios.

El papel de las agencias de publicidad ha sido analizado desde varios aspectos, así Sánchez (2002) estudia sus creencias y objetivos hacia la publicidad.

Los cambios en el medio televisión con la proliferación de canales han provocado y el consiguiente fraccionamiento de las audiencias, así Castellanos destaca la saturación y la ineficacia publicitaria (1994) y Rodríguez (1994) resalta la necesidad de la publicidad de adaptarse a un escenario radicalmente diferente.

Aunque la Licenciatura en la facultad de Comunicación recibe el nombre de Publicidad y Relaciones Públicas, nuestro área de marketing considera que esta denominación es excesivamente estrecha pues debido a la evolución que ha sufrido la comunicación comercial existen otras variables que deberían una importancia similar a las que recoge el nombre de la titulación como es el caso de la promoción de ventas o el emplazamiento de productos. 


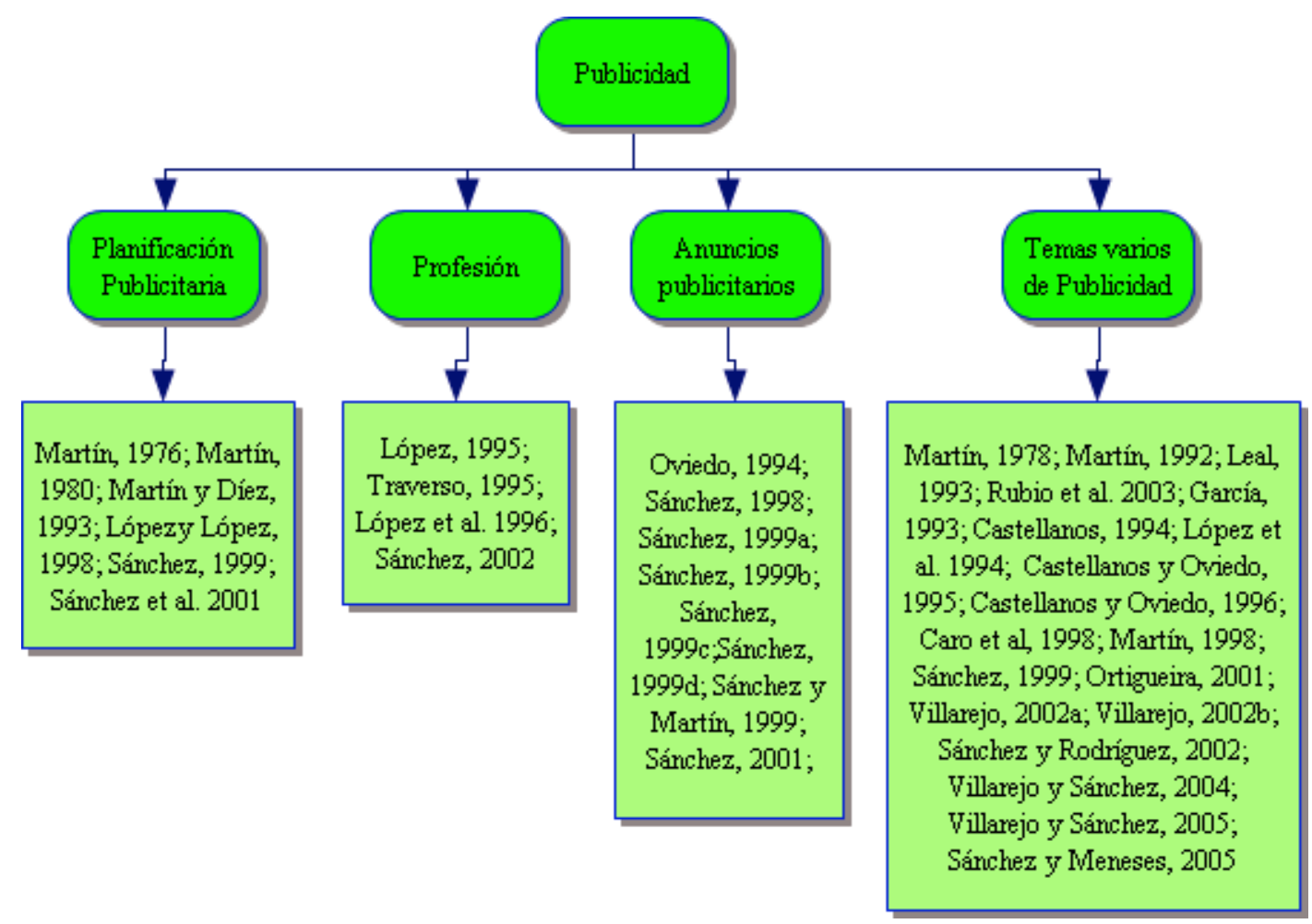

Ilustración 4: Investigaciones relacionadas con la Publicidad

\section{OTRAS VARIABLES DE COMUNICACIÓN COMERCIAL}

Caro y Rodríguez (1995), describen la realidad del marketing promocional en España detallando los medios, los instrumentos y los objetivos que persiguen. Sánchez y Rodríguez (2003) describen la variable promoción y Caro y Rodríguez (2004) tras desarrollar un estudio longitudinal de las promociones de ventas diarias, razonan el futuro de la comunicación comercial. Sanz y Ortega (2004) se centran en la promoción de áreas comerciales.

El emplazamiento de productos o product placement es otra de las variables de comunicación comercial que ha ganado en importancia en los últimos años.

Son los profesores Rodríguez y Caro (1999) los que desarrollan un trabajo pionero y proponen dotar de identidad propia al emplazamiento de productos, desmarcándolo de la errónea proposición de algunos autores que le deban el tratamiento de una forma ilícita de publicidad. Posteriormente se subraya la conexión entre el cine y la comunicación comercial (Rodríguez y Caro, 2001) y las mutuas ventajas que pueden alcanzarse al implantar el emplazamiento de productos en la estrategia de comunicación comercial. Caro y Rodríguez (2001) también subrayan la posibilidad de usar el emplazamiento de productos en nuevos soportes como es el caso del comic. 
La comunicación comercial es una de las cuatro variables del marketing-mix y engloba a la publicidad y a las relaciones públicas, por ello, el manual de planificación publicitara evoluciona a Comunicaciones de Marketing: Planificación y Control (Sánchez, Diez y Martín 2001) y varios profesores del departamento colaboran en el manual Estrategias y Técnicas de Comunicación (Salvador et al. 2007)

Varios son los trabajos que han analizado esta variable considerando la comunicación comercial como un todo: Villarejo y Sánchez (2004), resaltan los efectos de la comunicación comercial sobre el valor de marca y Garrido, Caro y Rodríguez (1998) detallan su uso en el la industria vinícola.

Las nuevas tecnologías también han ocupado un atraído nuestro interés en distintos trabajos, así Revilla y Domínguez (1998) observan las ferias virtuales y su implicación en la comunicación internacional. Martín (1998) estudiando la publicidad en internet plantea un estudio comparativo entre España y Portugal y Ortigueira y Vázquez (1998) ven a Internet como variable de comunicación.

Sánchez, Villarejo y Martin (2009) plantean los efectos moderados del sexo en la calidad de la relación y la lealtad de los proveedores de los servicios de internet.

Sin lugar a dudas la principal contribución de los profesores del departamento de Administración de Empresas y Marketing ha sido la de dotar a la publicidad en particular y a la comunicación comercial en general del rango estratégico que requieren, dándole sentido únicamente en el marco de una estrategia de marketing de índole superior. Aunque alguien pueda pensar lo contrario, la publicidad no es un fin sino un medio, que ha de perseguir objetivos claros, que ha de asumir la responsabilidad de sus resultados, que debe responder ante los anunciantes y demostrarles que su inversión, que no gasto, en publicidad ha sido rentable. 


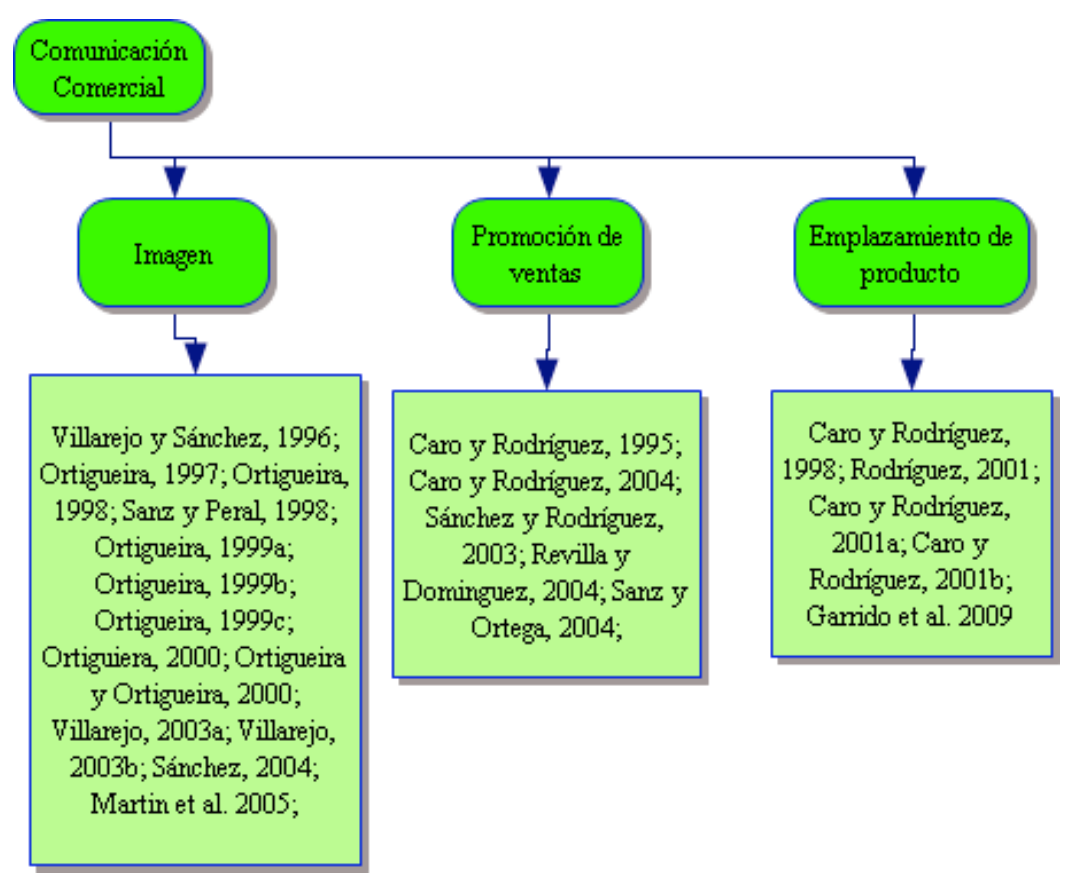

Ilustración 5: Investigaciones relacionadas con la comunicación comercial

\section{CONCLUSIONES}

La investigación relacionada con la comunicación por parte de los profesores del Departamento de Administración de Empresas y Marketing ha sido muy intensa. Los temas estudiados a lo largo de estos 20 años han sido muy variados tanto desde el área de Organización de Empresas como desde el área de Marketing. En la primera destacan los estudios relacionados con las empresas de comunicación, mientras que en la segunda llama la atención los estudios ligados a la Publicidad. También queremos destacar el hecho de que los profesores que han estado adscritos a la Facultad de Comunicación han publicado casi el $50 \%$ de los trabajos (a pesar de no suponer ni el $10 \%$ de los profesores del departamento). Esto indica la relación que existe entre el proceso de enseñanza-aprendizaje y la investigación. Se investiga sobre lo que se enseña y se enseña sobre lo que se investiga.

Hay que mencionar el caso de profesores como Caro, Rodríguez y Villarejo que realizan investigaciones multidisciplinares con profesores de los departamentos de Periodismo y de Comunicación Audiovisual, Publicidad y Relaciones Públicas. Es un camino por el que hay que apostar para enriquecer los conocimientos sobre cualquiera de los temas de estudio relacionados con la comunicación. 
Esperemos que este tipo de colaboraciones siga dando sus frutos en los próximos años. 


\section{BIBLIOGRAFÍA CITADA}

Alves, M. D. R. (2005): La Dirección de los Recursos Humanos y las Nuevas Tecnologías de la Información y la Comunicación (Formación a Distancia y e-Trabajo). Administración de Empresas y Comercialización e Investigación de Mercados (Marketing).Tesis doctoral dirigida por González, M.

Barroso, C. y Cossío, F. J. (1998): "El Proceso de Reclutamiento de la Fuerza de Venta: una Investigación Empírica de las Ofertas de Empleo en Prensa". Revista Europea de Dirección y Economía de la Empresa. Vol. 7. Núm. 1. Pp. 95-110.

Barroso, C.; Martín, E. y Martín, D. (2005): “Análisis de la Influencia de la Imagen en la Cadena de Lealtad en un Mercado Heterogéneo". Cities in Competition. New Trends in Marketing Management : Selected Papers From the XV Spanish-Portuguese Meeting of Scientific Management : Seville (Spain), February 2nd and 4th.

Calvo, A. y Criado, F. (2001): "Benchmarking en Procesos de Comunicación Interna: una Aplicación en Instituciones Universitarias". La Empresa Deslocalizada. Las Palmas de Gran Canarias. Marreo Hernández. Vol. 2.. Pp. 493-500.

Caro, F. J. (1996): "La Arquitectura de la Empresa: Un Elemento al Servicio de la Comunicación Integral". Questiones Publicitarias. Revista Internacional de Comunicación y Publicidad. Núm. 5. Pp. 75-86.

Caro, F. J. (2001): Comunicación. Administración y Dirección. Madrid. MC Graw-Hill. Vol. 1.Pp. 159-179.

Caro, F. J. (2002): Efectos de la Innovación y el Cambio en la Gestión y Organización de Empresas Informativas. Administración de Empresas y Comercialización e Investigación de Mercados ( Marketing) Tesis Doctoral dirigida por Diez, E. P.

Caro, F. J. y Casanueva, C. (1993): "Situación de la Comunicación Interna en las Empresas Españolas". Actas VII Congreso Nacional y III Congreso Hispano Francés de la Asociación Europea de Dirección y Economía de la Empresa. Córdoba. Etea. Num. 7. Pp. 1-2.

Caro, F. J. y Orti, A. M. (2008): Empresa Informativa. Sevilla. Secretariado de Recursos Audiovisuales y Nuevas Tecnologías de la Universidad de Sevilla.

Caro, F. J. y Rodríguez, C. J. (1995): "El Marketing Promocional en España: Medios, Instrumentos y Objetivos que Persigue". La Innovación en la Empresa. Toledo, Guarnizo García, J. Pp. 2319-2334.

Caro, F. J. y Rodríguez, C. J. (1997): "La Enseñanza de la Administración de Empresas Informativas a Través del Cine". Revista de Enseñanza Universitaria. Pp. 177-185.

Caro, F. J. y Rodríguez, C. J. (1998):" Mejora de la Enseñanza Universitaria a Través de Soportes Audiovisuales”. Revista de Enseñanza Universitaria. Pp. 261-274.

Caro, F. J. y Rodríguez, C. J. (2001a): El Emplazamiento de Productos en el Cómic. Comic, Comunicación y Cultura. Sevilla. Fernández Gómez y Otros. Pp. 119-130.

Caro, F. J. y Rodríguez, C. J. (2001b): "El Emplazamiento de Productos Como Variable Estratégica en la Mezcla de Comunicación”. El Management en el Próximo Milenio. Lepe (Huelva). Departamento de Administración de Empresas y Marketing Universidad de Sevilla. Pp. 175-181.

Caro, F. J. y Rodríguez, C. J. (2004): "Estudio Longitudinal de las Promociones en Prensa Diaria”. El Futuro de la Comunicación. Sevilla, Universidad de Sevilla. Vol. 1. Pp. 201-215.

Caro, F. J. y Rodríguez, C.J. (1998): "El Product-Placement: una Nueva Variable en el Mix de Comunicación”. Revista Do Instituto Superior Politécnico Portucalense. Vol. 2. Pp. 441-450. 
Caro, F. J.; Garrido, M.; Rodríguez, C. J. yVillarejo, A. F. (1998): "La Comunicación Comercial Como Instrumento de la Industria Vinícola en su Aproximación al Publico Joven". XIX Jornadas de Viticultura y Enología de Tierra de Barros. Jornadas de Viticultura y Enología de Tierra de Barros. Almendralejo. Cultural Santa Ana. Centro Universitario Almendralejo. Núm. 19. Pag. 525-532.

Caro, F.J. (1996): El uso de la prensa como soporte pedagógico en la enseñanza universitaria. IV Jornadas de Comunicación Social "Educación, Medios de Comunicación y Consumo". Sevilla, 20-27 noviembre

Caro, F.J. (2002): Cambio e Innovación en la Empresa Informativa. Universidad de SevillaGrupo de Investigación Gestión de la Innovación, la Calidad y el Cambio.

Caro, F.J. (2007a): Gestión de Empresas Informativas. Madrid. MC Graw-Hill.

Caro, F.J. (2007b): “ABC de Sevilla 2029: El Año del Centenario”. ABC de Sevilla, un Diario y una Ciudad. Sevilla. Universidad de Sevilla. Vol. 1. Pp. 321-341.

Caro, F.J. (2008): Organización y Gestión de Empresas de Servicios. Sevilla. Secretariado de Recursos Audiovisuales y Nuevas Tecnologías de la Universidad de Sevilla.

Caro, F.J. y García, J. (1996): Los Roles del Directivo y Sistemas de Dirección. Alta Dirección. $\mathrm{n}^{\circ} 201$, pp. 60-68.

Caro, F.J. y Jiménez, G. (2005a): La formación empresarial de los estudiantes de Periodismo. VII Foro Universitario de Investigación en Comunicación. Madrid.

Caro, F.J. y Jiménez, G. (2005b): El mercado de trabajo de los periodistas. VII Foro Universitario de Investigación en Comunicación.Madrid.

Caro, F.J. y Jiménez, G. (2006a): "Periodistas: el Acceso al Mercado Laboral". Ámbitos. Revista Andaluza de Comunicación. Pp. 313-334.

Caro, F.J. y Jiménez, G. (2006b): "Tendencias de Cambio en las Empresas Informativas". Global Media Journal en Español. Vol. 3. Núm. 5. pp. 12-20

Caro, F.J. y Oviedo, A. (1996): "La Industria de la Comunicación. Sus Estrategias de Crecimiento en la Economía Globalizada". La Empresa en una Economía Globalizada retos y Cambios. Granada. Teodoro Luque Martínez. Pp. 83-96.

Caro, F.J. y Rodríguez, C.J. (1995): "La Información Como Producto". Información y Ciencia. Pag. 11-19. Sevilla. Equipo de Investigación de Análisis y Técnica de la Información.

Caro, F.J.; García, M.M. y Rodríguez, C.J. (2007b): "Percepción de las Habilidades y las Capacidades de la Mujer en las Tareas de Dirección". Revista Andaluza de Comunicación. Núm. 016. pp. 395-407.

Caro, F.J.; García, M.M.; Rodríguez, C.J. y Jiménez, G. (2007a): "Percepción de las Habilidades y Aceptación de las Mujeres Directivas en las Empresas de Comunicación". Zer Revista de Estudios de la Comunicación de la Universidad del País Vasco. Vol. 12. Núm. 22, pp. 253-266.

Caro, F.J.; Rodriguez, C.J.; Garcia, M.M. y (2008): "The Influence of Gender on Entrepreneurial Activity in the Sector of the Press". Entrepreneurship and Small Business in International Aspect. Brno, Czech. Brno University of Technology. Pp. 20-35

Caro, F:J. (2008): "La Responsabilidad Social Corporativa en los Medios de Comunicación: el Caso de el País". Investigar la Comunicación. Santiago de Compostela. Ae-IC. Pp.1-10.

Casanueva, C.; Ganaza, J. D.; Espasandin, F. (2004): "Las Nuevas Tecnologías de la Información y la Comunicación en la Economía Social Andaluza". Ciriec-España. Revista de Economía Pública, Social y Cooperativa. Núm. 49. Pp. 193-215. 
Casanueva, C.; Rufino, J. I.; Periañez, R. y Caro, F. J. (2000a): “Análisis de los Resultados de la Utilización del Cine en la Enseñanza de la Gestión de Empresas." Revista de Enseñanza Universitaria. Núm. EXTR. Pp. 217-231.

Casanueva, C.; Rufino, J. I.; Periañez, R. y Caro, F. J. (2000b): “Aproximación Participativa a la Gestión de Empresas Por Medio de Proyecciones y Análisis de Casos". Materiales para la Calidad. Sevilla. Instituto de Ciencias de la Educación de la Universidad de Sevilla. Vol 2. Pp. 167-180.

Castellanos, M. (1994): Saturación e Ineficacia Publicitaria en la Televisión. VI Encuentros de Profesores Universitarios de Marketing. Madrid, ESIC. 1994. Pp. 191-217.

Castellanos, M. y Oviedo, A, (1996): "La Inversión Publicitaria Como Motor de la Economía". Administrando para la Sociedad. Sevilla. Diputación Provincial de Sevilla. Pp. 407-418.

Castellanos, M. y Carvajal, E. (1999): "La Campaña de Comunicación Institucional en el Proceso de Transición al Euro". El Management en el Próximo Milenio. Lepe (Huelva). Departamento de Administración de Empresas y Marketing. Universidad de Sevilla. Pp 107115.

Castellanos, M. y Oviedo, A. (1995): "Economic Recession, Advertising Recession". Conference on Business Development in Central and Eastern Europe and Its Implications for the Economic Integration of Ceec in the Wider Europe. Brno, República Checa. PC-Dir Real. Pp. 371-381.

Castellanos, M.; Rosa, I. M.; Caro, F. J. y Oviedo, A. (2008): Medición de la Imagen y Repercusión Socioeconómica de Eventos Deportivos. Informes, Estudios, Trabajos y Dictámenes.

Criado, F.; Rufino, J. I.; Roldán, J.L.; Moreno, A.M. y Villegas M. M. (1996): "La Gestión de la Comunicación Como Recurso Estratégico". Gestión y Organización de Empresas Comerciales. Sevilla. Gráficas Minerva. Pp. 85-114.

Del Barrio, S.; Rodríguez, I.; Bigné, J. E. ; Küster I; Rodríguez del Bosque, I. A.; Alet, J.; García, M. M. ; Rodríguez, J. ; Sánchez M. J. y Suárez A. (2007): Estrategias y Técnicas de Comunicación. Barcelona. Editorial Uoc, S.L.

Díaz, M. C. y Picón, A. (2001): "El Comportamiento del Buscador de Empleo Frente a las Ofertas Insertas en los Anuncios en Prensa". La Empresa Deslocalizada. Las Palmas de Gran Canarias. Marreo Hernández. Vol. 2. Pp. 145-155.

García del Junco, J. (1992): "Estrategia de Imagen Global en las Empresas Españolas: un Reto para el 92". ESIC-Market Revista Internacional de Economía y Empresa. Pp. 137-152.

García del Junco, J. (1993): "Aspectos de la Publicidad en la Cee”. ESIC-Market Revista Internacional de Economía y Empresa. Pp. 124-137.

García, J. (2004): "Aplicación de las Nuevas Tecnologías de la Información y de la Comunicación". Gerir a Competitividade Regional Num Mundo Globalizado. Ponta Delgado, Universidad de las Azores. Portugal. Pp. 345-370.

Garcia, J. y Dutschke, G. (2005): "The Future Use of Internet As Comunication, Information Selling Channel, By Fast Moving Consumer Good Companies". Innovations and Technology Projects and Operations Management in the City Development. Sevilla. Universidad de Sevilla - Mergablum, S.L. Pp. 472-487.

García, R. y Revilla, M. A. (1999): "La Comunicación Internacional”. La Internacionalización de las Empresas: Casos Prácticos. Sevilla. Atril S.L. Pp. 183-201.

Jiménez, G. y Caro, F.J. (2007): "Identidad, responsabilidad y corporate branding en la industria textil española: el caso de Zara". III Congreso Internacional Investigación y RRPP. Universidad de Sevilla, Sevilla.

Landa, F. J. y Diez, E. C. (1996): Merchandising: Teoría y Práctica. Madrid. Pirámide S.A. 
Leal F. J. (1993): "La Publicidad : la Influencia del Factor Legal en su Gestión". Administración de Empresas y Comercialización e Investigación de Mercados (Marketing).Tesis Doctoral dirigida por Diez de Castro E. C.

López Bonilla L. M., López Bonilla J. M. (1998): "Subconjuntos Borrosos y Planificación de Soportes Publicitarios: Determinación del Alcance y la Distribución de Contactos". Uncertainty Logics: Applications in Economics and Management. Sigef 98. Lausanne (Suiza). H.N. Teodorescu, J. Gil Aluja y D. Mlynek. Pag. 62-81

López, M. L. (1995): "La Formación Universitaria: un Producto Ofertado a las Empresas. El Caso de la Licenciatura de Publicidad y Rrpp y el Sector Publicitario en la Localidad de Sevilla”. VII Jornadas Hispano-Lusas de Gestión Científica. Jornadas Hispano Lusas de Gestión Cientifica. Sevilla. Diputación de Sevilla. Servicio de Publicaciones. Num. 7. Pp. 182197.

López, M. L.; Traverso, J. y López, L: (1996): "Reposicionamiento Estratégico del Licenciado en Publicidad y Relaciones Publicas en el Mercado Laboral. Questiones Publicitarias. Revista Internacional de Comunicación y Publicidad. Núm. 5. Pp. 23-36.

López, M: L.; Casillas, J. C. y Villegas, M. M. (1994): La Publicidad Conjunta: un Caso Emergente de Cooperación. VI Encuentro de Profesores Universitarios de Marketing. Madrid. ESIC. 1994. Pp. 247-257.

Martín Armario, E. y Diez de Castro, E. C. (1993): Planificación Publicitaria. Madrid. Pirámide S.A. 480.

Martín, D. (1998): "La Publicidad en Internet: un Estudio Comparativo Entre Portugal y España". VIII Jornadas Luso-Espanholas de Gestao Cientifica. Jornadas Luso-Espanholas de Gestao Cientifica. Oporto, Portugal. Universidad Portugalense. Num. 8.

Martín, D.; Barroso, C. y Martín, E. (2005): “Análisis de la Influencia de la Imagen en la Cadena de Lealtad en un Mercado Heterogéneo". Cities in Competition. XV Spanish-Portuguese Meeting of Scientific Management. Sevilla. Universidad de Sevilla. Núm. 15.

Martín, E. (1980): La Gestión Publicitaria. Madrid. Pirámide S.A.

Martín, E. (1976): Aplicaciones Económicas del Análisis Factorial de Correspondencias a Operaciones de Control Publicitario. I Coloquios Sobre Temas Empresariales. I Coloquios Sobre Temas Empresariales (1). La Rábida. Publicaciones del C.U.R. Núm. 1. Pp. 1-40.

Martín, E. (1978): "Determinación del Montante Satisfactorio del Presupuesto Publicitario". Revista de Economía y Empresa. Vol. ENERO. Núm. 1. Pp. 65-81.

Martín, E. (1992): La Globalización del Mercado Europeo y la Gestión Publicitaria. Tempori Serviendum. Granada. Milladorio. Pp. 213-228.

Martín, E. y Landa, F. J. (1991): La Manipulación de la Comunicación Como Estrategia de Apoyo a la Puesta en Escena del Marketing Politico: un Análisis Teórico Real. V Gongreso de AEDEM (5). La Coruña. Num. 5.

Martín, E. y Landa, F. J. (1992): La Manipulación de la Comunicación en Marketing Político. Tendencias Actuales en Administración de Empresas. Departamento de Administración de Empresas y Marketing. Universidad de Sevilla. Vol. 1. Pp. 133-141.

Ortigueira M. (2001): "La Comunicación en las Relaciones de Intercambio: un Modelo Normativo". ESIC-Market Revista Internacional de Economía y Empresa. Núm. 108. Pp. 73-92.

Ortigueira, M. (1997): Marketing Publico: Imagen de España y de Otros Países en un Marco Internacional, Administración de Empresas y Comercialización e Investigación de Mercados ( Marketing).Tesis doctoral dirigida por Martín, E.

Ortigueira, M. (1998): "Marketing Público: la Imagen de Portugal en Brasil". Revista Do Instituto Superior Politécnico Portucalense. Vol. 1. Pp. 345-358. 
Ortigueira, M. (1999a): “A Imaxe de Arxentina”. Revista Galega de Economía. Vol. 8. Núm. 1. Pp. 141-164.

Ortigueira, M. (1999b): "La Imagen de Portugal en España”. Revista de Economía,

Ortigueira, M. (1999c):"La Imagen de un País y su Gobierno: la Imagen de Alemania. Dirección y Organización". Revista de Dirección, Organización y Administración de Empresas. Núm. 22. Pp. 72-84 .

Ortigueira, M. y Ortigueira, M. (2000): Las Clases o Servicios Docentes Universitarios: Reflexiones en Torno a su Calidad y la Calidad de su Imagen. Materiales para la Calidad. Sevilla. Instituto de Ciencias de la Educación de la Universidad de Sevilla. Vol. 500. Pp. 143158.

Ortigueira, M. y Ortigueira, M. (2001): El Sistema Sanitario Público, Sus Servicios y Sus Responsables: Creación y Pilotaje de su Imagen. Cuadernos de Gestión. Pp. 67-103.

Ortigueira, M. y Vázquez, R. (2000): Internet Como Variable de Comunicación: Diferentes Implicaciones. Gestao XXI. Futuro e Perspectivas. X Jornadas Luso-Espanholas de Gestão Científica. Vilamoura (Portugal). Universidade Do Algarve (Portugal). Pp. 167-176.

Ortigueira, M.(2000): "La Política Pública de Imagen: la Imagen de Francia". Revista Europea de Dirección y Economía de la Empresa. Vol. 9. Núm. 2, pp. 167-192.

Oviedo, A. (1994): El Rol de la Mujer en los Anuncios Publicitarios. VI Encuentros de Profesores Universitarios de Marketing. Madrid, ESIC. 1994. Pp. 259-263.

Oviedo, A. y Quirós, F. J. (1997): "El Anuncio de Reclutamiento en Prensa: un Estudio del Caso Español (1912-1996)". XI Congreso Nacional. VII Congreso Hispano-Francés. Asociación Europea de Dirección y Economía de la Empresa (AEDEM). Lleida. Manuel Ruiz González. Pp. 471-478.

Oviedo, A.; Rodríguez-Bobada, J. y Sánchez, M. J. (2001): "La Investigación Publicitaria en España en los Años 1990-1998”. Nom Idem Iterum, Semper Novum. Sevilla, Edición Digital @ Tres. Pp. 111-121

Revilla, M. A. (2001): Imagen Ética de los Vendedores en la Relación de Compraventa: Situación del Sector del Automóvil en la Comunidad Autónoma Andaluza, Administración de Empresas y Comercialización e Investigación de Mercados( Marketing) Tesis doctoral dirigida por Ortigueira, $M$.

Revilla, M. A. (2004): La Ética en la Venta desde la Óptica del Consumidor: la Imagen Ética de los Vendedores en la Relación de Compraventa. Libro de Ponencias. XVI Encuentro de Profesores Universitarios de Marketing. Epum. XVI Encuentro de Profesores Universitarios de Marketing. Alicante. ESIC. Núm 16, pp. 847-867.

Revilla, M. A. y Domínguez de la Concha-Castañeda, M. (1998): Las Ferias Virtuales, un Nuevo Instrumento de Comunicación Internacional. Las Ciencias, las Técnicas y el Arte Aplicadas al Marketing. Madrid. Universidad Castilla la Mancha. Pp. 67-79.

Revilla, M. A. y Gallego, M. A. (2006): Impacto de la Filosofia Moral Personal del Consumidor en la Imagen Ética de los Vendedores. XVI Jornadas Luso-Espanholas Gestao Cientifica. Evora. Fundación Luís de Molina. Pp. 61-73.

Rodríguez, C. J. (2001): "El Emplazamiento de Productos: la Integración Entre el Cine y la Comunicación Comercial". Comunicación, Historia y Sociedad : Homenaje a Alfonso Braojos. Sevilla. Universidad de Sevilla - Ayuntamiento de Sevilla. Pp. 671-682.

Rodríguez-Bobada, J. y Castellanos, M. (1999): "El Mercado Televisivo en el Próximo Milenio". El Management en el Próximo Milenio. Lepe (Huelva). Departamento de Administración de Empresas y Marketing .Universidad de Sevilla. Pp 157-166. 
Rubio Picon, C. (2000): "Los Anuncios de Empleo en Prensa Como Forma Publicitaria: la Relevancia de la Redacción”. Questiones Publicitarias. Revista Internacional de Comunicación y Publicidad. Vol. 8. Pag. 109-119.

Rubio, C. y Díaz, M. C. (2000): "Un Estudio del Diseño de los Anuncios en Prensa de Ofertas de Empleo". Gestao XXI: Futuro e Perspectivas. Vilamoura (Portugal). Pp. 211-226.

Rubio. C.; García, J. y Rodríguez C. J. (1993): “¿Aspectos de la Publicidad en la C.E.?”. ESICMarket Revista Internacional de Economía y Empresa. Núm. 84. Pp. 125-136.

Sánchez Franco, M. J. (1999): Eficacia Publicitaria. Teoría y Práctica. Madrid. MC Graw-Hill.

Sánchez, M. J. (1998): La Cultura: un Análisis de su Influencia Sobre la Duración del Anuncio Publicitario. X Encuentro de Profesores Universitarios de Marketing. Madrid. ESIC. Pp. 105117.

Sánchez, M. J. (1999): Apuntes de Publicidad. Sevilla. Minerva.

Sánchez, M. J. (1999a): "La Importancia de la Dimensión Hedónico-Placentera Sobre la Estrategia de Ejecución del Anuncio Entre Consumidores Escasamente Implicados". Revista Europea de Dirección y Economía de la Empresa. Pp. 161-174.

Sánchez, M. J. (1999b): "Relación Entre el Recuerdo y la Actitud Hacia el Anuncio Publicitario: un Análisis Empírico". ESIC-Market Revista Internacional de Economía y Empresa. Pp. 105-126.

Sánchez, M. J. (1999c): 15 Segundos Vs. 30 Segundos: la Influencia de la Motivación y la Familiaridad del Receptor en la Elección de la Duración del Anuncio. Investigación y Marketing. Núm. 64. Pp. 53-56.

Sánchez, M. J. (1999d): El Color en el Anuncio Publicitario. La Gestión de la Diversidad. Logroño. AEDEM. Pp. 429-440.

Sánchez, M. J. (2001): "La Posición del Anuncio en la Pausa Publicitaria: Recomendaciones al Anunciante Ante Entornos Publicitarios Excedentes en Información". Revista Europea de Dirección y Economía de la Empresa. Vol. 10. Núm. 2. Pp. 133-148.

Sánchez, M. J. (2002): Objetivos y Creencias Hacia la Publicidad: la Visión de las Agencias. Investigación y Marketing. Núm. 7. Pp. 14-22.

Sánchez, M. J. y Martín, F. A. (1999): Los Clutters Publicitarios: Investigación Empírica Sobre los Anuncios en Televisión y su Nivel de Competencia. El Management en el Próximo Milenio. Lepe (Huelva). Departamento de Administración de Empresas y Marketing. Universidad de Sevilla. Pp. 209-222.

Sánchez, M. J. y Meneses, C. (2005): Fundamentos de Publicidad. Sevilla, Edición Digital @ tres.

Sánchez, M. J. y Rodríguez-Bobada, J. (2002): La Universidad: Sus Creencias Hacia la Publicidad. XIV Encuentro de Profesores Universitarios de Marketing. Granada. ESIC. Núm. 14. Pp. 219-241.

Sánchez, M. J. y Rodríguez-Bobada, J. (2003): Promoción de Ventas. Estrategias y Técnicas de Comunicación. Barcelona, Universitat Oberta de Catalunya.

Sánchez, M. J.; Diez, E. C. y Martín, E. (2001): Comunicaciones de Marketing : Planificación y Control. Madrid. Pirámide S.A.

Sánchez. M. (2004): Diseño de un Modelo de Imagen Básica para la Gestión de la Identidad Corporativa de las Organizaciones no Gubernamentales, Administración de Empresas y Comercialización e Investigación de Mercados ( Marketing). Tesis Doctoral dirigida por García del Junco, J. 
Sanz, B. y Peral, B. (1998): Factores Determinantes de la Imagen de las Grandes Superficies Especializadas. Business in a Colaborative World. Estambul. Turquia. Muzaffer Bodur. Pp. 419-426.

Sanz. B. y Ortega, J. (2004): "Promoción de Áreas Comerciales a Través de la Constitución de Centros Comerciales Abiertos". El Futuro de la Comunicación. Sevilla, Universidad de Sevilla. Vol. 1. Pp. 1-15.

Traverso, J. (1995): “Capital Humano: su Formación Universitaria y la Adecuación a las Necesidades Empresariales. El Caso de la Licenciatura de Publicidad y RR.PP. de la Universidad de Sevilla”. VII Jornadas Hispano-Lusas de Gestión Cientifica. Jornadas Hispano Lusas de Gestión Científica. Sevilla. Diputación de Sevilla. Servicio de Publicaciones. Núm. 7. Pp. 45-60.

Traverso, J. (1996): "Comunicación Interpretativa: Variable Clave en el Marketing Mix de las Empresas de Turismo Rural”. Estudios Turísticos. Núm.130. Pp 37-50.

Traverso, J. (2002): Análisis de la Imagen Interna de la Institución Universitartia. Estudio de la Imagen Interna de la Universidad de Sevilla, 2002, Administración de Empresas y Comercialización e Investigación de Mercados (Marketing). Tesis Doctoral dirigida por Diez de Castro, E. P.

Traverso, J. (2002): Estudio de la Imagen Interna de la Institución Universitaria. La Imagen Interna de la Universidad de Sevilla. Sevilla. Edición Digital @ Tres.

Traverso, J. (2004): "La Imagen de los Destinos Turísticos Elemento Básico en su Desarrollo Estratégico". Congreso Internacional "Patrimonio, Desarrollo Rural y Turismo en el Siglo XXI. Congreso Internacional Patrimonio, Desarrollo Rural y Turismo en el Siglo XXI. Osuna (Sevilla). Universidad de Sevilla. Núm. 1, pp. 621-634.

Traverso, J. (2005): "Imagen Interna de la Institución Universitaria. Modelo para el Personal de Administración y Servicios". Revista de Economía y Empresa. Vol. 23. Núm. 54-55, pp. 95112.

Traverso, J. (2007): "Modelo para la Obtención de la Imagen de un Destino Turístico". Estudios Turísticos. Vol. 1. Núm. 174, pp. 33-48.

Traverso, J. y García J. (1997): “Análisis de los Sistemas de Dirección en las Empresas de Comunicación. Estudio Comparativo Según Medios". La Empresa Creando Futuro. AEDEM. Lleida. Manuel Ruiz González. Universidad de Lleida. Pp. 901-911.

Traverso, J. y Román, M. L. (2006): "Desarrollo de la Imagen de un Destino Turístico. Modelo de Gestión". Faces. Vol. 25. Pp. 12-27.

Traverso, J. y Román, M. L. (2007): “Análisis de la Imagen Interna de Institución Universitaria. Construcción de un Modelo para la Obtención de la Imagen Bajo la Perspectiva del Alumno". Investigaciones Europeas de Dirección y Economía de la Empresa. Vol. 13. Núm. 1, pp. 231246.

Villarejo, A. F. (2002a): "El Esfuerzo Publicitario Como Antecedente del Valor de Marca en el Mercado de Bienes de Conveniencia". Investigación y Marketing. Núm. 75, pp. 6-15.

Villarejo, A. F. (2002b): La Percepción del Esfuerzo Publicitario Como Antecedente en el Modelo de Medición del Valor de Marca. Contrastación Empírica para el Caso de un Producto Duradero. XIV Encuentro de Profesores Universitarios de Marketing. Madrid, ESIC. Pp. 279291.

Villarejo, A. F. (2003a): "La Influencia de la Imagen del Establecimiento Comercial en la Determinación del Valor de Marca de los Bienes de Conveniencia". Gestión Científica Empresarial : Temas de Investigación Actuales. La Coruna. Netbiblo. Pp. 175-188.

Villarejo, A. F. (2003b): La Influencia de la Imagen del Establecimiento Comercial en la Determinación del Valor de Marca de los Bienes de Conveniencia. XIII Jornadas Hispano- 
Lusas de Gestión Científica. Jornadas Hispano-Lusas de Gestión Científica. Lugo, Universidad de Santiago de Compostela. Num. 13, pp. 31-41.

Villarejo, A. F. y Sánchez, M. J. (1996): "La Imagen de Marca: una Aproximación a las Estrategias de Internacionalización". La Empresa en una Economía Globalizada: Retos y Cambios. Congreso Nacional y V Hispano Francés de la Asociación Europea de Dirección y Economía de la Empresa. Granada. Edinford. Núm. 9, pp. 731-741.

Villarejo, A. F. y Sánchez, M. J. (2004): Los Efectos de la Comunicación Comercial Sobre el Valor de Marca de los Bienes Duraderos. XIV Jornadas Luso Espanholas de Gestào Científica. Universidad Dos Açores. Universidad Dos Açores. Pp 2015-2025.

Villarejo, A. F. y Sánchez, M. J. (2005): Marketing Communication As Precedent for Brand Equity. a Look Into the Markets of Lasting Goods. 4th European Marketing Trends Congress. 4th European Marketing Trends Congress. Paris. Escp. Pp. 1-18.

Villarejo, A. F.; Sánchez, M. J. y Rondan, F. J. (2005): “Antecedentes de la Notoriedad del Nombre en la Determinación de la Imagen de Marca. Una Visión desde un Producto de Gran Consumo". El Comportamiento de la Empresa Ante Entornos Dinámicos. XIX Congreso Nacional y XV Congreso Hispano-Francés de AEDEM. Vitoria-Gasteiz. AEDEM. Pp. 19-28. 